\title{
No effect of lunar cycle on the energy or macronutrient intakes of adults
}

\author{
R. L. Bates and S. Whybrow \\ Rowett Institute, University of Aberdeen, Aberdeen AB25 2ZD
}

Many of the purported lunar rhythms in human behaviours are now disproved, but an increase in energy and alcohol intake around the time of the full moon, reported by de Castro \& Pearcey ${ }^{(1)}$, remains untested in an independent sample. This study tested the hypothesis that energy and macronutrient intakes, as reported by adults, differ significantly between the new moon and the full moon.

A pre-study power calculation suggested that a sample of 56 would give the power to detect an $8 \%$ difference in energy intake, as reported previously ${ }^{(1)}$, with a power of 0.8 and a $0.05 \%$ significance level. Eighty-eight adults $(25$ males, 63 females, median age $34 y$ completed two online 24 -hour recalls ${ }^{(2)}$. One was completed during a new moon and the other during a full moon, with the order being counterbalanced over the study. Intakes were completed on the same, or following, day of the week, which were Tuesdays, Wednesdays or Thursdays, and over a six-week period during summertime in the UK. Participants were not informed of the true purpose of the study. Energy and macronutrient intakes were calculated and compared, and accounting for order of completion of the recalls and menstrual cycle.

Mean daily energy intake was $7759 \mathrm{~kJ}$ and $8461 \mathrm{~kJ}$ during the new moon and full moon respectively $(\mathrm{P}=0.168)$. There was no significant difference in the contribution of macronutrients to energy intake between the new moon and full moon $(\mathrm{P}>0.226)$. There was no significant effect of order of completion of the recalls, or phase of menstrual cycle.

Contrary to the findings of the one previous study that reported on the effects of lunar rhythms on meal intakes, the results of this study, which used a more powerful study design, suggest that lunar rhythms in energy and macronutrient intakes are not present in the eating behaviours of adults.

1. de Castro J \& Pearcey S (1995) Physiol Behav 57, 439-444.

2. Rowland M, Poliakov I, Christie S et al. (2016) Newcastle University \& Food Standards Scotland 